\title{
Preliminary Aerodynamic Measurements from a Magnetic Suspension and Balance System in a Low-Speed Wind Tunnel
}

\author{
Mark Schoenenberger ${ }^{1}$, David E. Cox ${ }^{2}$, Timothy D. Schott ${ }^{3}$, Anne I. Mackenzie ${ }^{4}$, Otoniel Ramirez ${ }^{5}$, \\ NASA Langley Research Center, Hampton, VA 23666 \\ Colin P. Britcher ${ }^{6}$, Cameron Neill ${ }^{7}$, Michelle Weinmann ${ }^{7}$, Desiree Johnson ${ }^{7}$ \\ Old Dominion University, Norfolk, VA 23529
}

\begin{abstract}
An existing Magnetic Suspension and Balance System (MSBS) has been restored to operation in a small (octagonal test section, 6-inches across flats) low-speed wind tunnel as the first phase of a project to develop new dynamic test capabilities for planetary entry capsules at supersonic speeds. The MSBS and associated wind tunnel were originally designed and built at MIT and were subsequently relocated to NASA Langley Research Center. NASA ceased operations of the system in the late 1990s and it was transferred to Old Dominion University where it had been mothballed until the present work started in 2015. All-new position sensor electronics, electromagnet power supplies, digital controller, and other hardware have been developed, leading to more efficient and reliable operation. Important features of the new hardware will be briefly described. Wind tunnel testing has commenced, focusing first on static aerodynamics of re-entry capsule shapes. Some preliminary results will be reviewed, including comparison to reference data. The proposed extension to dynamic stability testing, using a variety of free-to-pitch approaches will be described. Finally, the preliminary design of the all-new Phase II (supersonic) system will be briefly outlined.
\end{abstract}

\section{Nomenclature}

$\mathrm{B}_{\mathrm{x}, \mathrm{y}, \mathrm{z}} \quad$ Magnetic flux density in the $\mathrm{x}, \mathrm{y}$ and $\mathrm{z}$ directions (Tesla)

$\mathrm{B}_{\mathrm{xx}} \quad$ Gradient of flux density in the axial (tunnel flow) direction

d Capsule diameter

$\mathrm{C}_{\mathrm{D}} \quad$ Drag coefficient, $\mathrm{Fx} /(\mathrm{qs})$

$\mathrm{F}_{\mathrm{x}} \quad$ Force in the $\mathrm{x}$ direction

$\vec{M} \quad$ Magnetization vector $(\mathrm{A} / \mathrm{m})$

$\mathrm{M}_{\mathrm{x}, \mathrm{y}, \mathrm{z}} \quad$ Components of the magnetization vector along the $\mathrm{x}, \mathrm{y}$ and $\mathrm{z}$ axes

q Dynamic pressure

$\mathrm{Re}_{\mathrm{d}} \quad$ Reynolds number based on model diameter: density*velocity*diameter/(kinematic viscosity)

$\mathrm{S} \quad$ Reference area (area of circle with diameter of wind tunnel model)

$\vec{T} \quad$ Torque vector

$\mathrm{T}_{\mathrm{x}, \mathrm{y}, \mathrm{z}} \quad$ Torque components about the $\mathrm{x}, \mathrm{y}$ and $\mathrm{z}$ axes

$\mathrm{x}, \mathrm{y}, \mathrm{z} \quad$ Tunnel axes: $+\mathrm{x}=$ upstream, $+\mathrm{y}=$ right when looking upstream,$+\mathrm{z}=$ down

\footnotetext{
${ }^{1}$ Research Engineer, Atmospheric Flight and Entry Systems Branch, Senior Member AIAA

${ }^{2}$ Research Engineer, Dynamic Systems and Controls Branch

${ }^{3}$ Electronics Engineer, Remote Sensing Branch

${ }^{4}$ Research Electrical Engineer, Electromagnetics and Sensors Branch

${ }^{5}$ Pathways appointee, Atmospheric Flight and Entry Systems Branch, Student Member AIAA

${ }^{6}$ Professor of Aerospace Engineering, AIAA Associate Fellow

${ }^{7}$ Graduate student, Department of Mechanical and Aerospace Engineering, Student Member AIAA
} 


\section{Introduction and Overview}

Contemporary planetary entry capsules are characterized by low length-to-diameter ratios, such as the Galileo and Pioneer Venus entry capsules [1], the Stardust comet sample return capsule [2], the Mars Exploration Rover entry vehicles (MER) [3], the Mars Science Laboratory (MSL) [4], the Orion Multi-Purpose Crew Vehicle (MPCV) [5], and others. Some examples are shown in Figure 1. Aerodynamic and aerothermodynamic analysis has typically proceeded with a mix of computational and experimental work. A wide range of experimental facilities are brought to bear, with specific topics of interest including static aerodynamics (i.e. lift and drag), heat transfer rates to the heat shield, and dynamic stability, all over a wide range of Mach numbers [6]. Dynamic stability at low supersonic Mach numbers has emerged as a key area of concern for these entry vehicles as proper orientation is critical for successful parachute deployment.

Experimental dynamic stability testing at low supersonic Mach numbers has relied to a great extent on aeroballistic ranges [7], although this approach tends to yield a limited quantity of data and the availability of facilities in the U.S. has recently declined. Free-to-oscillate and forced-oscillation wind tunnel testing has also been used to measure entry vehicle dynamic stability characteristics as well as vertical spin tunnel testing (at low subsonic conditions). Understanding the observed differences between test data collected using these different techniques is challenging. The Reynolds number and reduced frequency design spaces in wind tunnels often do not overlap with the test conditions in aeroballistic ranges. Furthermore, the sting effects in traditional dynamic testing are difficult to quantify and may be significant for blunt bodies, where dynamics are dominated by separated wake flows. Computational capabilities for the prediction of blunt body dynamic stability continue to improve, but to date have not performed well in predicting oscillation growth of dynamically unstable vehicles as observed in supersonic ballistic ranges. To help reconcile data using different test techniques and to provide more validation data for the development of unsteady computational codes, NASA is investigating the possibility of constructing a new supersonic wind tunnel test facility, relying on the use of a Magnetic Suspension and Balance System (MSBS) to provide model motion capability, hence the ability to conduct dynamic stability testing. As will be discussed further, this could resemble a traditional forced oscillation rig, albeit with support interference eliminated, a traditional freeto-pitch rig, or some hybrid or variation thereof. Cameras will track the oscillations and small motions of the magnetically suspended model relative to the freestream flow. The dynamic aerodynamic coefficients will be determined by fitting a trajectory through the observed motions, much as is done in a ballistic range. Unlike in a ballistic range, flow conditions can be held constant when suspending the model in a wind tunnel, allowing cameras to measure much more data at a given condition. It is hoped that MSBS test capabilities can help the understanding of the differences between traditional tunnel testing and ballistic range test results and provide high-rate, accurate trajectory data to help improve computational codes.

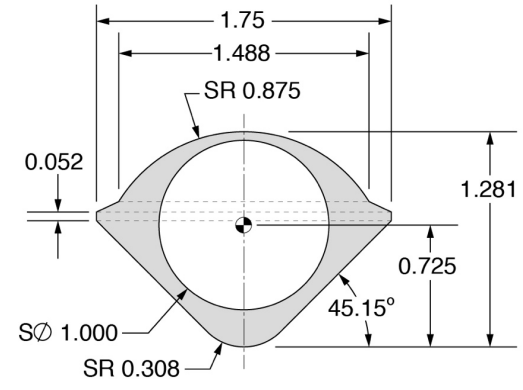

GALILEO Entry Probe

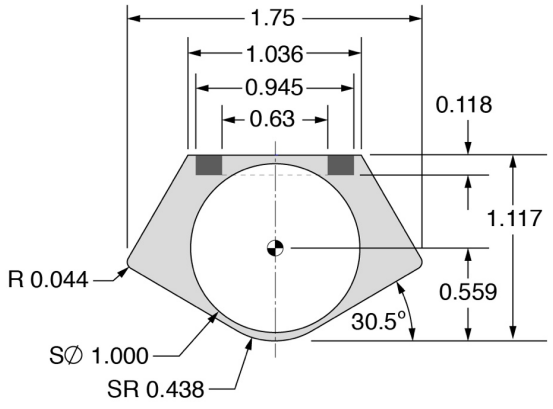

STARDUST

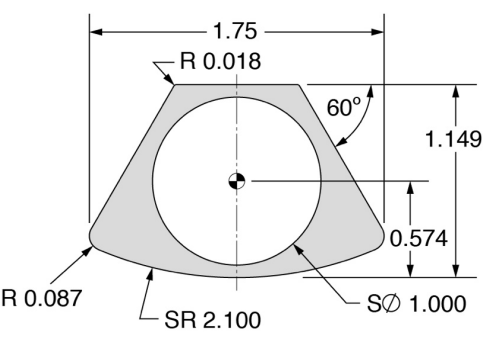

ORION

(Symmetric)

Figure 1 - Examples of Planetary Entry Capsule Geometries

Galileo (left), Stardust (center), Orion (right)

All shown with MSBS magnetic cores, dimensions as tested, in inches

\section{Magnetic Suspension and Balance System (MSBS) Overview}

Magnetic Suspension and Balance Systems were first demonstrated in the 1950's, with almost immediate application to supersonic wind tunnels [8]. Over the subsequent decades, a number of government research organizations and universities pursued research programs, although activity declined in the late 1990's as it became 
clear that a MSBS for a large transonic wind tunnel would be prohibitively expensive. Over the last few years, active technology development programs have been pursued at Tohoku University in Japan, where three systems of varying size are operational $[9,10]$, and in Korea [11]. In addition, at least one system has been in routine use for aerodynamic measurements at Oxford University, in the U.K. [12].

MSBSs rely on a magnetic core embedded within the model, either magnetizable material ("iron") or permanent magnet. In the former case the core is magnetized by a stroing uniform applied field, usual along the tunnel axis. Moments can be generated about transverse axes by applying transverse fields. Applied field gradients then produce forces along all three axes. All fields and field gradients are produced by electromagnets external to the wind tunnel test section. Systems of this type are always open-loop unstable in at least one degree of freedom, so position and attitude is sensed and the electromagnet currents controlled in real-time via a feedback controller. Once stable suspenson/levitation is achieved, calibration of electromagnet currents can provide real-time information on magnetic forces and moments.

One of the promises of MSBSs for wind tunnel testing has been the possibility of dynamic stability testing using forced-oscillation or free-oscillation techniques. Forced-oscillation testing was conducted by MIT and the University of Southampton, including axisymmetric and winged models. Magnus force testing with axisymmetric models spinning at high speeds about their axes was also accomplished [13-16]. In these tests, models with axially magnetized cores (iron at MIT and permanent magnets at Southampton) were controlled in five degrees of freedom. Oscillation was created by injection of trajectory command signals into the feedback controllers. More recently, Tohoku University has successfully implemented the forced oscillation technique in their $0.1 \mathrm{~m}$ MSBS in a lowspeed wind tunnel [17].

At least two groups (Princeton University and the University of Virginia) explored free-oscillation testing, where the model contained a spherical, freely magnetized iron core, such that only magnetic forces could be generated $[18,19]$. The spherical core was embedded in a non-magnetic (sometimes also non-conducting) aerodynamic shell, with pitch/yaw oscillations initiated by a variety of means. Although restricted to axisymmetric shapes, three degreeof-freedom levitation using a spherical magnetic core was shown to be practical and was employed at supersonic speeds. Dynamic stability measurements were reported by the UVa group [19]. It should be noted that both projects relied on vertically oriented wind tunnels, such that axial symmetry is maintained and the gravitational force on the model directly opposes drag. This configuration, although advantageous, is not thought to be a fundamental requirement for success.

\section{Project Overview}

Due to the low length-to-diameter ratio of the candidate geometries, coupled with familiarity with free-flight trajectory-based testing (i.e. ballistic ranges), it was decided to pursue the free-oscillation approach in this project [20]. For blunt bodies, where the supersonic dynamic stability is driven primarily by separated wake flows, the dynamic moments are very small compared to the static aerodynamic moments. It can be difficult to extract the small dynamic aerodynamic moments from moments imparted by forced oscillations. However, reversion to a forced-oscillation approach with either freely magnetized or permanent magnet model cores remains possible in the future. For now, free-to-oscillate test methods are being pursued. The project was defined in two phases:

i. Establish a proof-of-concept subsonic system using hardware (principally an electromagnet array, Electromagnetic Position Sensor (EPS) "cage", and wind tunnel) from a previous MSBS project. This constitutes Phase I and is the main focus of this paper.

ii. Design and construct an all-new system for a modified test section leg of an existing supersonic wind tunnel. The preliminary design of such a system will be briefly reviewed.

This free-to-oscillate approach assumes that a spherical model core will be free to rotate, while the MSBS system actively suspends the model within the test section. Ideally this setup would operate much like a vertical spin tunnel (without the constraint that the model weight be balanced by its drag), or a stingless free-to-oscillate rig in a traditional wind tunnel. This approach assumes a core material with a low coercive force, where the magnetization vector in the core easily align with the magnetizing field as the model rotates, providing little resistance to rotation. Early materials tested to date, including the nickel-iron core used in the Galileo model show more resistance to rotation than expected. Additional materials will be tested and free-to-oscillate testing may be realized as originally envisioned, but alternative approaches for achiving free-to-pitch oscillations in the tunnel are being explored which will be described below. The preliminary tests reported here focused on validating static force measurements against historical data and did not require models to oscillate freely in the tunnel. 


\section{MIT/NASA 6-inch Magnetic Suspension and Balance System}

In the mid- to late-1960's a team at MIT designed and developed a sophisticated MSBS that was mainly used in a low-speed wind tunnel, although was actually designed for supersonic operation [21]. The MSBS and low-speed wind tunnel were relocated to NASA Langley Research Center in the early 1980's and operated for a decade or so [22]. Following an extended "retirement", the electromagnet assembly (illustrated in Figures 2 and 3), the electromagnetic position sensor (EPS) system, and the low-speed wind tunnel form the basis of the Phase I proof-of-concept system. The electromagnets are water-cooled, hollowconductor copper, with iron yokes for the transverse gradient coils. The design inherently provides some separation of magnetic field and gradient components between coils, such that not all coils are needed for the proposed system.

The EPS system is not currently used in any other MSBS. It operates as a multi-axis linear variable differential transducer (LVDT) [21,2325 ] over a cubic volume, roughly 3 inches on a side. The EPS has been selected as the firstchoice position sensor in the proposed system since it offers relative insensitivity to model shape. The EPS system detects the presence of a magnetizable and/or an electrically conducting volume which modulates the reception of a reference signal broadcast by excitation coils. This modulation is related to the physical position of the metallic core within the EPS sensor. One set of excitation and three sets of receiver coils provide measurements along three orthogonal axes. Once calibrated the EPS measures the translational position of a spherical model core. If it is later found that both position and orientation information is required, the EPS system has additional coils which can determine the angular position of a slender asymmetric

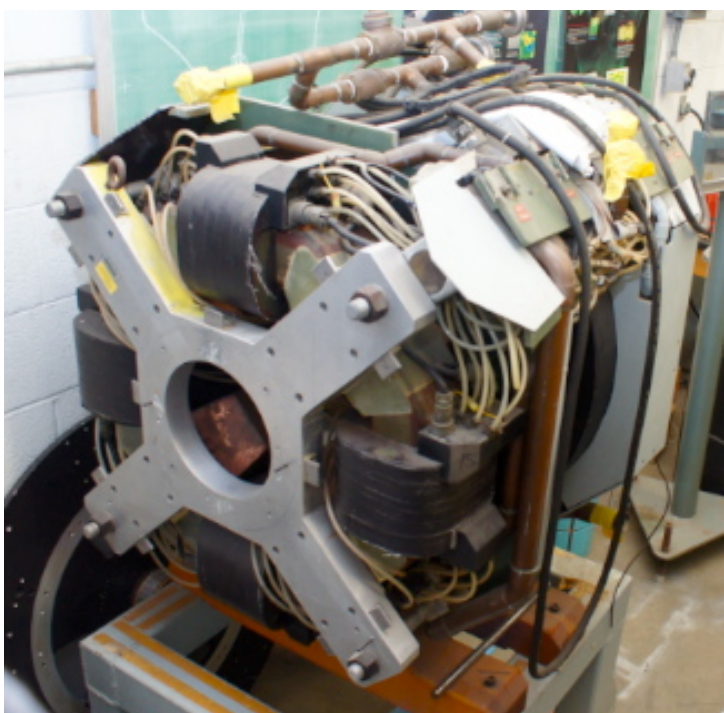

Figure 2 - MIT "6-inch" MSBS Electromagnet Assembly

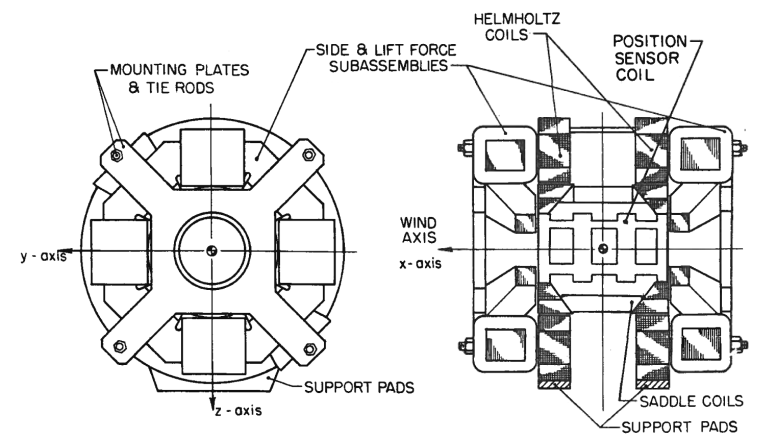

Figure 3 - MIT MSBS Electromagnet Configuration. core. This analog system returns continuous feedback to the controller at very high rates. The MIT system is the only known successful implementation of the EPS approach in a MSBS.

In contrast, camera-based optical systems are more problematic when observing low L/D models that may be oscillating up to large angles of attack. An optical system requires the determination of the model centroid based on external markings. Typically reference lines or a series of dots are tracked. Reference markers may be misinterpreted when a blunt capsule oscillates up to large angles and more robust tracking methods require significant real-time computational capabilities. Preliminary analysis of conditions in a candidate supersonic tunnel, where model rotation and translation rates can be very high, it was found that state-of-the-art camera-based motion tracking systems do not track model position and orientation at sufficient data rates.

To more accurately define the potential operational capabilities and to facilitate development of control algorithms, a mathematical model of the electromagnet array has been developed in COMSOL, with some details previously reported [26,27].

\section{A. Summary of New Hardware}

A $30 \mathrm{~kW}$ DC power supply feeds the magnetizing coils (up to $120 \mathrm{~A}$ continuous), with controlled currents for levitation derived from 4 switching-mode power amplifiers, in turn fed from a single $45 \mathrm{~kW}$ DC power supply. Two 
power amplifiers are connected in parallel to provide up to 120A continuous in the axial (drag) coil. The other 2 provide up to $60 \mathrm{~A}$ continuous to the vertical (lift) and lateral (side) coils respectively. The EPS electronics have been re-built with various design improvements. The control systems are now all-digital (for the first time with this particular MSBS), based on a Speedgoat ${ }^{\mathrm{TM}}$ target machine, programmed via MATLAB/Simulink.

The wind tunnel drive system was also updated, with a new AC motor with variable-frequency drive and PCbased control and data acquisition system. The MSBS was originally designed for use with a supersonic wind tunnel, but actually spent most of its operational life installed in the subsonic wind tunnel, depicted in Figure 4 . The test section is an octagonal cross-section, nominally 6-1/4 inches across flats. The contraction ratio exceeds 10:1, with four screens. The centrifugal blower induces flows up to around Mach 0.5. Since recommissioning, the speed has been held to below Mach 0.2 .

The updated system became operational in late 2017. Since then, system development has continued and preliminary aerodynamic measurements carried out. Capsule shapes have been constructed from 3D printed shells, each with a maximum diameter of $1.75 \mathrm{in}(44.5 \mathrm{~mm})$, containing magnetic cores. The shells are made in two halves, split at the maximum diameter of the magnetic core and assembled around the cores with adhesive. The cores tested thus far have varied in both material choice and shape, as discussed more fully later.

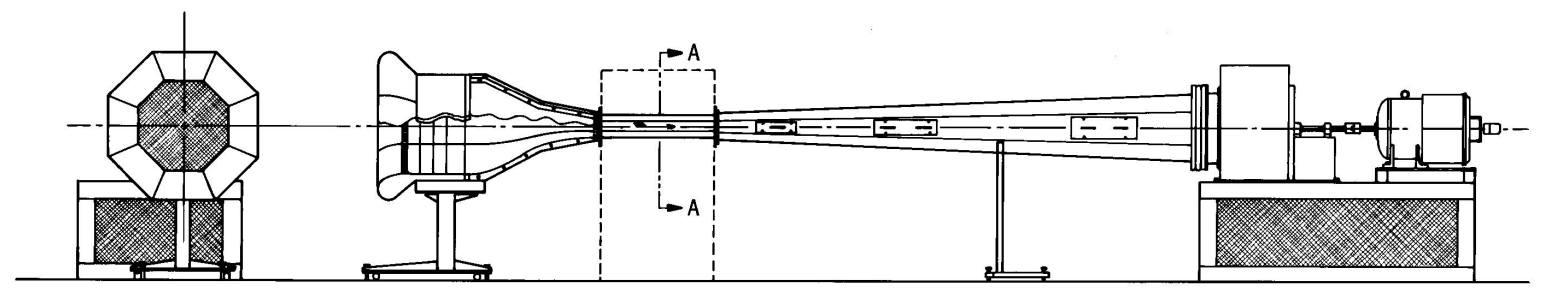

Figure 4 - The 6-inch Low Speed Wind Tunnel

\section{Preliminary Aerodynamic Testing}

As part of the system commissioning process, static aerodynamic measurements are being made on a selection of standard shapes and heritage entry vehicles. Initial results are focused on measuring drag and serve to identify any deficiencies in facility instrumentation, operational procedures and ultimately data quality. Force measurements with a MSBS rely on measurement of currents in the electromagnets and calibration of same against force. By using identical magnetic cores in a range of non-magnetic shells, the same force calibration is made applicable to multiple tests. The governing equation for drag coefficient measurements is as follows:

$$
F_{x}=M_{x} B_{x x} \mathrm{Vol}
$$

For a magnetically soft core, $M_{x}$ is induced by the magnetizing field, and will be approximately 3 times the strength of the magnetizing field, which is held constant. If the core is a permanent magnet, $M_{x}$ is fixed. Figure 5 shows two typical force calibrations, carried out by applying axial forces to the suspended magnetic core via a fine thread, pulley and weight pan. The fine thread is attached to the magnetic core and passed over a pulley so that calibrated masses may be hung. The masses placed on the weight pan exert a pulling force on the levitated models along the tunnel axis, in place of the aerodynamic drag forces seen during testing. The permanent magnet cores exhibit high linearity with the current sent to the magnetic coils that produce the axial flux density gradient, $B_{x x}$, whereas the magnetically soft core (NiFE sphere) appear slightly nonlinear. This is due to various effects, perhaps including small changes in magnetization as the axial gradient $B_{x x}$, becomes larger. The force pulling on the model during calibration is of course the product of the mass and the acceleration of gravity. The gravitational acceleration at sea level and the latitude of NASA Langley is $\left(9.79913 \mathrm{~m} / \mathrm{s}^{2}\right)$. Therefore, the force/amp coefficients for the NiFe and Neodymium balls are 0.0194 and 0.0611 Newtons/Amp respectively.

For the test data presented here, the drag data is determined solely by the measured current, calibrated as described above. All models were tested at small angles of attack. For larger angles of attack or models that produce lift, the currents providing vertical force and side force can be used to measure the aerodynamic forces acting along those axes. The current required to lift the mass of the model must be removed as a tare to determine the aerodynamic lift forces. The present configuration does not utilize any moment control of the model. Orthogonal high-speed video files will be post processed, deriving aerodynamic moments from the observed capsule 
oscillations. Small model motions can be accounted for in the video data as well. Future work will investigate a Kalman filter approach, where position and orientation information from cameras, EPS position information and measured currents will be used together to produce a best fit and determine a full set of aerodynamic coefficients. Again, in these preliminary tests, only the axial current is used for drag force measurement.
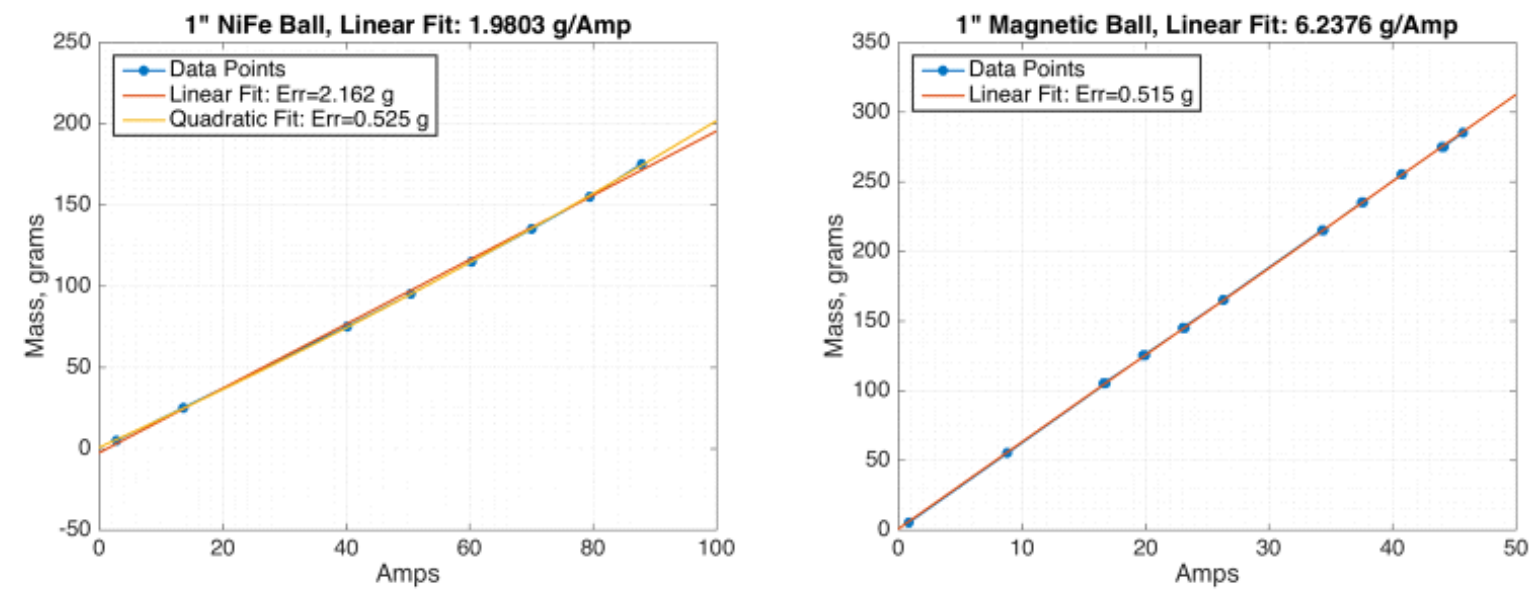

Figure 5 - Drag Force Calibration of Magnetically Soft Core (left) and Permanent Magnet Core (right) Calibration forces are the hung mass multiplied by the local gravitational acceleration.

The wind tunnel dynamic pressure is derived in the traditional way by measurements of the static pressure difference across the contraction section (contraction ratio $>10: 1$ ) with a correction factor derived from empty test section pitot measurements. Results are corrected for solid and wake blockage following classical formulae [28]. Corrections result in a reduction in drag coefficient for the tested re-entry shapes of about $3 \%$. No buoyancy correction is applied since the test section has a slight axial taper, resulting in a very low axial pressure gradient. Figure 6 shows the drag coefficient measured on a small sphere (a model core without an aerodynamic shell) and a sphere of the same diameter as the capsule models (a 3D printed shell with the same magnetic core). Reference $C_{D}$ values are derived from the curve fit presented by Mikhailov and Silva Freire [29], based on the experimental data by Roos and Willmarth [30]. Comparison to reference data appears satisfactory at this early stage of system development. Two points should be noted. First, the surface finish of the large sphere (a 3D printed aeroshell) was somewhat rough compared to the small sphere, which was relatively smooth (a bare magnetic core). Second, the magnitudes of forces at low tunnel speed are very low, leading to degraded precision.

Three capsule geometries have been tested for this work - Galileo, Stardust, and Orion, as shown previously in Figure 1. For each test run, the current readings were recorded before the tunnel was started to provide a wind-off
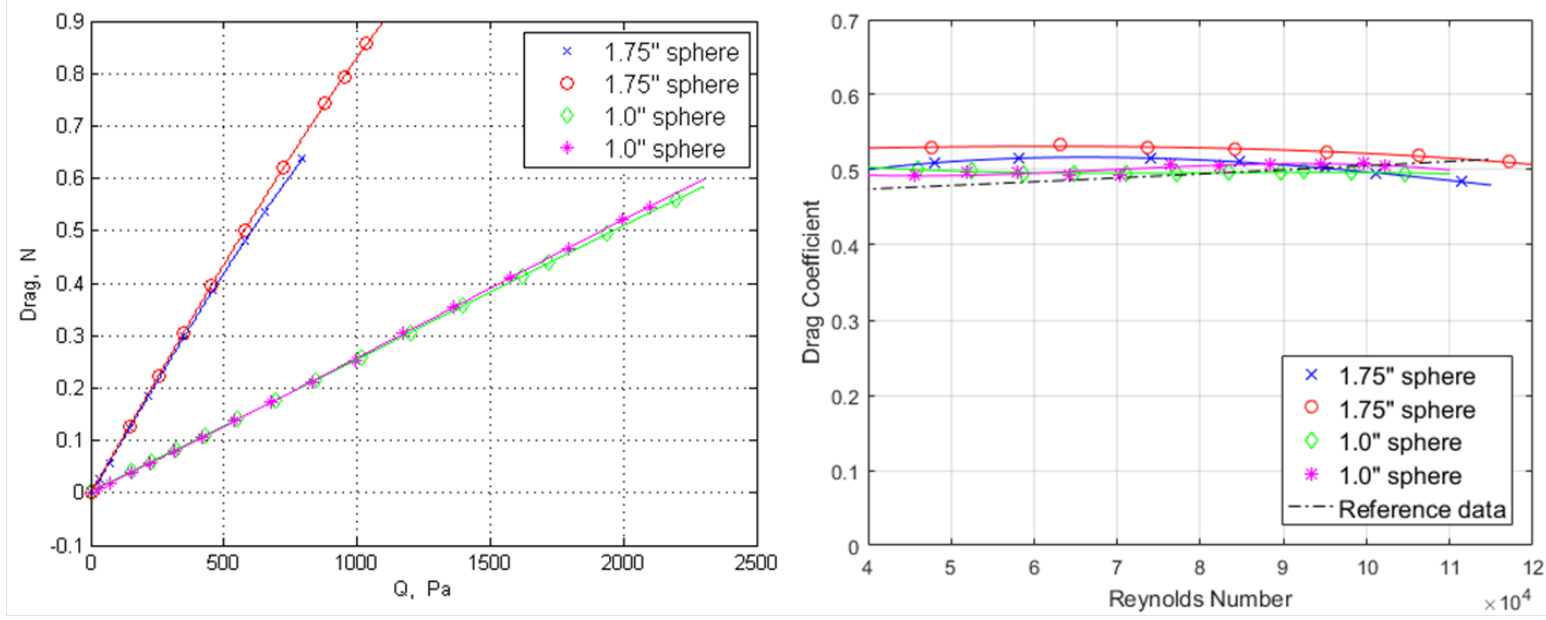

Figure 6 - Sphere Force versus $q$ and $C_{D}$ versus Re

American Institute of Aeronautics and Astronautics 
tare. The tunnel was then closed and tunnel speed was increased incrementally until aerodynamic forces overpowered the controller's response currents and the model departed from the null point in the tunnel. Typically the commanded currents were well below the limits of the power supplies. The controller used for these tests was designed to allow for capsule motions to occur without immediate high-frequency response. The intent is for the model to behave as if it were free flying within small volume in the tunnel test section. If the model deviates too far from the null point, the controller will actively return the model. As dynamic pressure increases, the model motions increase in amplitude and frequency. At the higher dynamic pressure, the controller does not respond quickly enough and the model can be lost down the tunnel. Refinements to the controller algorithm for higher speed testing should enable tests at higher dynamic pressures in the future. The test space for the data presented here is listed in Table 1.

Table 1 - Test Conditions for Drag Runs

\subsection{0 in Diameter Spheres}

\begin{tabular}{ll}
\hline Mach & $0-0.175$ \\
Dynamic Pressure & $0-2200 \mathrm{~Pa}$ \\
Red $_{\mathrm{d}}$ & $0-102,000$ \\
Velocity & $0-60 \mathrm{~m} / \mathrm{s}$ \\
\hline
\end{tabular}

\subsection{5 in. Diameter Models}

\begin{tabular}{ll}
\hline Mach & $0-0.128$ \\
Dynamic Pressure & $0-1175 \mathrm{~Pa}$ \\
Re $_{\mathrm{d}}$ & $0-131,000$ \\
Velocity & $0-44 \mathrm{~m} / \mathrm{s}$ \\
\hline
\end{tabular}

Galileo was tested with a magnetically soft spherical core, specifically a Nickel-Iron alloy resembling Carpenter 49. Stardust and Orion were tested with a spherical Neodymium-Iron-Boron (NdFeBo) core. Figure 7 shows drag force versus q and drag coefficient versus Reynolds number for two runs of the Orion shape. Drag coefficients are only computed at Reynolds numbers above 40,000 since forces are very small below this value $(q<110 \mathrm{~Pa}, \mathrm{D}<0.2 \mathrm{~N})$. Figure 8 shows a comparison of higher-q runs for all 3 shapes.
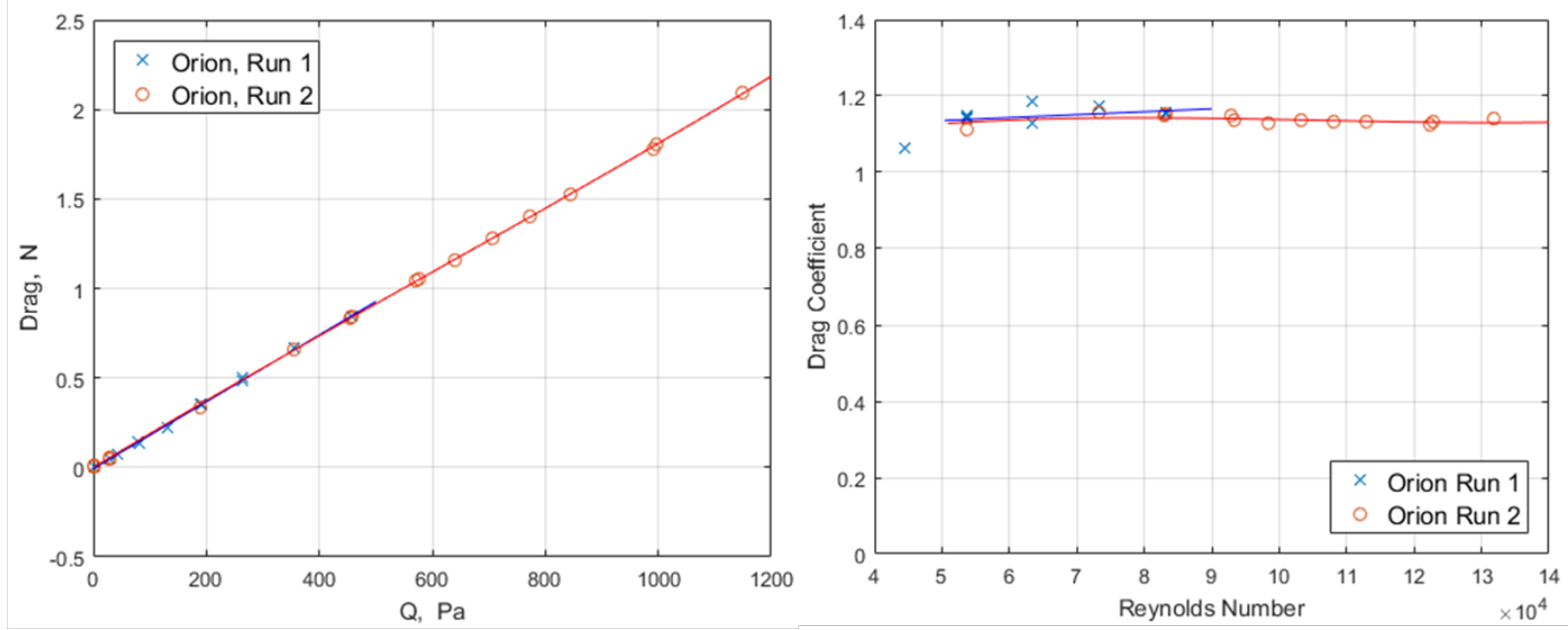

Figure 7 - Orion Run Comparison; Force versus $q$ and $C_{D}$ versus $R e$ 
The low speed drag coefficients of these shapes is expected to be relatively insensitive to backshell geometry, since this entire region is enveloped in the separated wake. Increasing bluntness of the forebody would be expected to result in increased drag coefficients, since the initial angle of departure of the shear layer from the maximum diameter becomes more normal to the flow, perhaps tending to increase the wake size. This trend is observed in the results presented.

Reference data from a variety of sources is also shown in Figure 8, all corresponding to historical tests conducted at substantially higher Reynolds numbers. Pioneer Venus data is shown for comparison with Galileo as the configurations are similar and there is more subsonic data available for Pioneer Venus. The somewhat higher drag coefficients shown in the current tests could be related to the Reynolds number disparity or to the relatively rough surface finish of the 3D printed model shells. Future work will test for Reynolds number effects with vertical spin tunnel models sized to match these subsonic test conditions and the conditions of the historical tests. It is also expected that improved controller performance will allow enable the models to be tested at higher Reynolds numbers in the magnetic suspension tunnel. Finally, smooth models fabricated with traditional machining technology will be tested to assess the roughness effects introduced by the surface quality of the 3D printed models. Figure 9 shows all geometries suspended in the test section prior to wind tunnel runs.

The apparent angle of attack of the capsule shapes is due to small C.G. offsets from the magnetic center (all cases), the resistance of the magnetization vector to realign with the magnetization field (Galileo) or initial misalignment of the permanent magnet core inside the aeroshell (Stardust and Orion). In the latter case, the magnetic cores were actually free to rotate inside the aeroshell, with a small gap between the cores and plastic outer shells filled with mineral oil. Imprecisions of the 3D printed shells resulted in residual friction between the core and shell which resisted the aerodynamic moments acting on the model. In all cases, the geometries are statically stable, so tend to realign to the velocity vector once sufficient dynamic pressure builds up. Measurement of capsule attitude in the tunnel using video data at the larger dynamic pressures showed that the capsules were incident to the freestream flow at angles varying from 0 to 5 degrees.

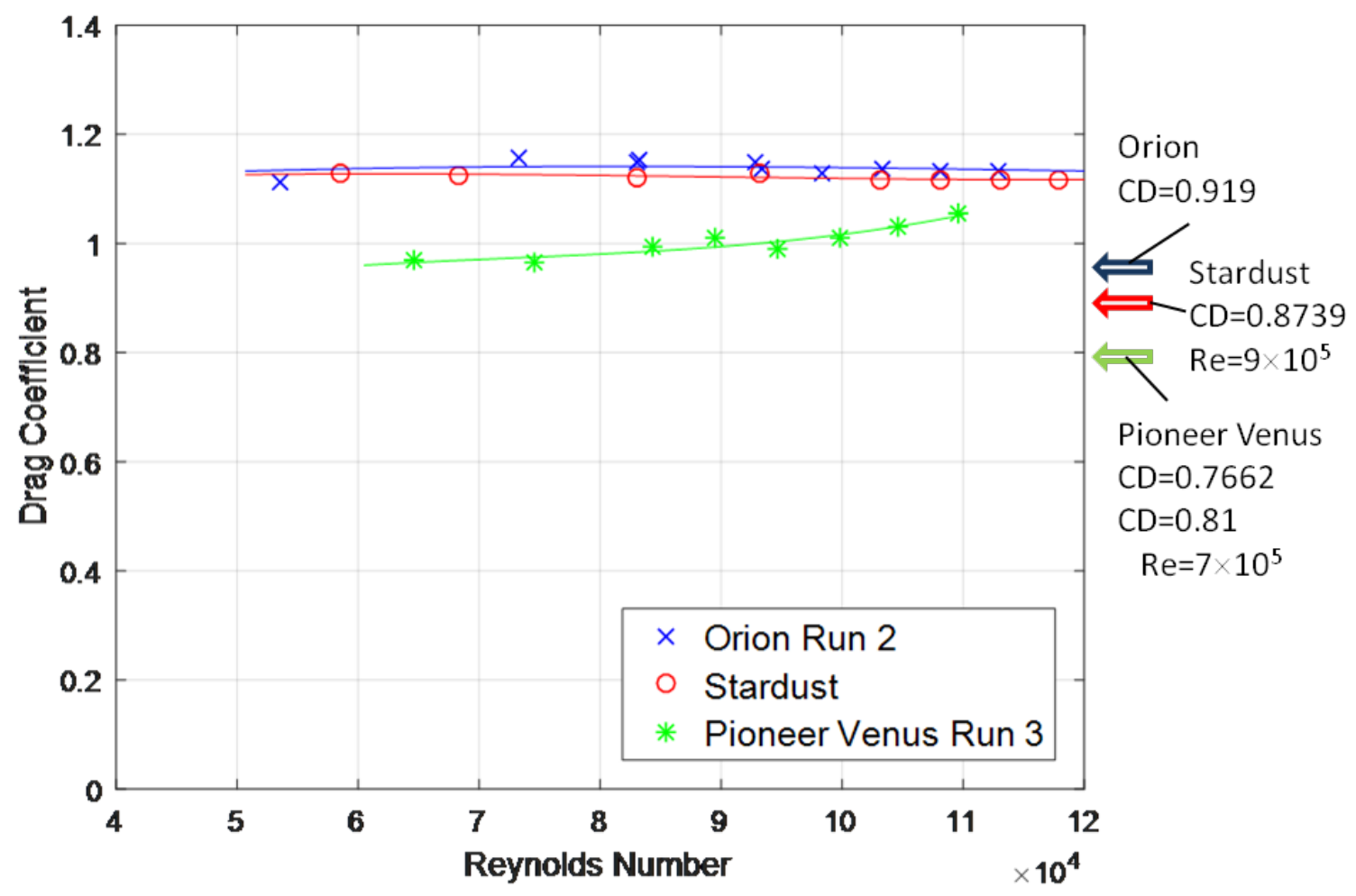

Figure 8 - Comparison of Orion, Stardust and Galileo Drag Coefficients 


\section{Phase II Overview}

\section{A. Facility Objectives}

The wind tunnel facility selected for accommodating the supersonic MSBS is the NASA Glenn $225 \mathrm{~cm}^{2}$ supersonic wind tunnel, which is a classical blowdown type, with interchangeable square and axisymmetric crosssectiontest sections, nominally, $15 \mathrm{~cm}$ across flats or $225 \mathrm{~cm}^{2}$ circular area. Octagonal test sections provide slightly more favorable interface to the MSBS, notably smaller diameter axial coils and would also allow for larger wind tunnel models before a tunnel unstart. The flat walls of an octagonal test section also provide for good optical access to the model. A design for a new Mach 2.5 nozzle incorporating an axisymmetric throat with a supersonic transition from square to octagonal has been completed and analyzed with CFD. A prototype will be built for experimental evaluation shortly. Preliminary assessments of facility requirements, notably electrical power and cooling water, have been conducted. An issue with supersonic operation of MSBSs is transient load during startup and shutdown, as the starting shock convects through the test section. Mechanical restraints have traditionally been used during tunnel start, however, recent work at Tohoku University has shown that a model can be suspended during an entire supersonic tunnel run without mechanical restraints [31].

The issue of the magnitude of forces expected on the suspended model, and the ratio of these to the model's weight is a critical concern for two reasons. The drag force must be countered by a combination of the magnetizing field and the axial gradient (drag) field, as discussed earlier. Previous calculations have indicated that the dynamic pressure capability of the 6-inch low-speed tunnel is lower but approaches the same order-of-magnitude of the
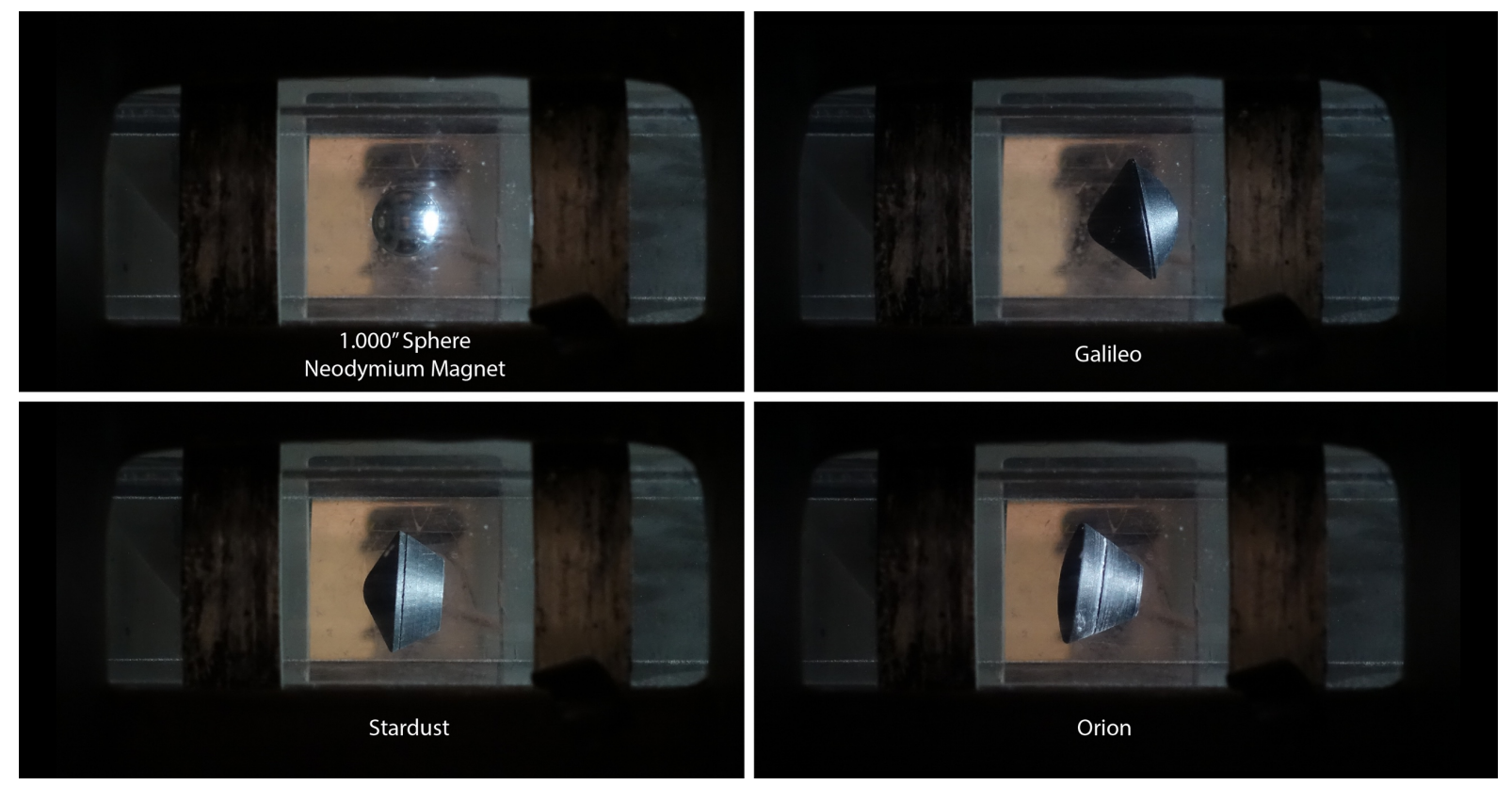

Figure 9 - Models in Suspension Prior to Tunnel Runs. Flow is Left-to-Right

dynamic pressures likely in the proposed supersonic facility. It is noted that minimizing stagnation pressure, even assuming atmospheric downstream pressure, is advantageous.

\section{B. System Configuration Options}

The original concept for dynamic stability testing was to implement suspension of a spherical model core, comprised of magnetically soft material (i.e. iron), controlled in 3 translational degrees of freedom, but with the model free to rotate in all other degrees of freedom. This concept was previously implemented by the University of Virginia [18] and Princeton University [19] with similar objectives, albeit with the wind tunnel axes in both examples aligned vertically. This approach requires that the magnetic core material has a low coercive force in order to avoid unwanted stiffness about the axial direction (the magnetizing field) and/or a tendency to exhibit a preferred orientation when suspended wind-off. Also, the material should have a relatively high resistivity, to avoid induced 
eddy currents during rotation of the magnetic core, which would lead to damping of the motion. Analysis and experimentation to quantify these effects and to identify the optimum material choice is continuing.

An unexpected offshoot of these studies had been a realization that permanent magnet cores can also be suspended under 3 degree-of-freedom control. By way of example, small spherical (as illustrated in this paper), or cylindrical permanent magnet cores (Neodymium-Iron-Boron material) have been embedded in aerodynamic shells and suspended with the magnetization vector along the wind tunnel axis. A weak axial "magnetizing" field then causes the core's magnetization vector to align with this field. This is successful for static testing at zero degrees angle of attack and could be extended to variable angles of attack by re-activating the existing "saddle" coils, which permit the re-orientation of the net magnetic field at the system centroid. Importantly, it can be seen from the equation for magnetic torque on a dipole (shown below) that there is no mechanism for generation of torque about the magnetization axis, likely also low damping of rotations, since the resistivity of NdFeBo core materials is naturally high. This opens the possibility of transversely magnetized cores, free to pitch about the axis of magnetization, which is being explored further.

$$
\begin{gathered}
\vec{T}=(\vec{M} \times \vec{B}) \quad \text { (governing equation) } \\
T_{x}=0 ; \quad T_{y}=-M_{x} B_{z} ; \quad T_{z}=M_{x} B_{y} \quad \text { (axial magnetization) } \\
T_{x}=M_{y} B_{z} ; \quad T_{y}=0 ; \quad T_{z}=-M_{y} B_{x} \quad \text { (transverse (y) magnetization) }
\end{gathered}
$$

\section{Summary and Conclusions}

A project aimed at developing a new test capability for dynamic stability testing of planetary entry capsules at low supersonic Mach numbers has been outlined. A Phase I proof-of-concept system MSBS is operational, leveraging an existing electromagnet array, electromagnetic position sensing system and low-speed wind tunnel. Preliminary aerodynamic testing has been completed and low angle-of-attack drag data have been measured on a range of heritage entry vehicle shapes. The drag coefficients measured on these vehicles are higher than historical data, although the Reynolds numbers are much lower than those at which the historical tests were conducted. The surface roughness of the 3D printed models may also affect the drag. Future tests will investigate the impact of these effects.

No insurmountable obstacles have been identified for the Phase II supersonic MSBS system. The coercive forces inherent to the magnetic cores tested thus far are too great to achieve satisfactory "free-to-oscillate" behavior in the tunnel. Other core materials will be investigated as will the possibility of aligning the magnetizing field transverse to the tunnel flow to create a $1 \mathrm{D}$ free-to-oscillate test configuration. Work will continue, improving the controller algorithm and developing the motion capture system with the objective of capturing sting-free capsule dynamics. Such tests will enable an understanding of blunt body dynamics and to provide validation data for unsteady CFD development. The data measured so far and the lessons learned during commissioning activities indicate that MSBS can be a useful test technique for supersonic dynamic testing.

\section{Acknowledgments}

This work was supported by NASA Langley Research Center, Entry Systems Modeling Project, a part of NASA's Game Changing Development Program, under activity NNL09AA00A, \#2A78, \#2B28, and \#201001 awarded to the National Institute of Aerospace. The NASA monitor is Mark Schoenenberger, Atmospheric Flight and Entry Systems Branch.

\section{References}

1. P.F. Intrieri, D. B. Kirk, "High-Speed Aerodynamics of Several Blunt-Cone Configurations," 24 $4^{\text {th }}$ AIAA Aerospace Sciences Meeting, January 1986. AIAA 86-0300.

2. R. Michletree, et. al., "Aerodynamics of Stardust Sample Return Capsule", $15^{\text {th }}$ AIAA Applied AerodynamicsConference, July 1997. AIAA 1997-2304

3. M. Schoenenberger, W. Hathaway, L. Yates, P. Desai, "Ballistic Range Testing of the Mars Exploration Rover Entry Capsule", 43rd AIAA Aerospace Sciences Meeting, January 2005. AIAA 2005-55

4. M. Schoenenberger, L. Yates, W. Hathaway, "Dynamic Stability Testing of the Mars Science Laboratory Entry Capsule", 41st AIAA Thermophysics Conference, June 2009. AIAA 2009-3917 
5. B. Bihari, et. Al., "Orion Capsule Handling Qualities for Atmospheric Entry", AIAA Guidance, Navigation and Control Conference, August 2011, AIAA 2011-6264

6. B.R. Hollis, et.al., "Entry, Descent and Landing Aerothermodynamics: NASA Langley Experimental Capabilities and Contributions", 52nd AIAA Aerospace Sciences Meeting, January 2014. AIAA 2014-1154

7. T.N. Canning, "Ballistic Range Technology", AGARDograph No.138, August 1970

8. M. Tournier, P. Laurenceau, "Suspension Magnetiqued'une Maquette en Soufflerie", La RechercheAeronautique \#59, July-August 1957, pp:21-27

9. H. Sawada, S. Obayashi, "A New 1-m Magnetic Suspension and Balance System for the Low Turbulence Wind Tunnel at IFS", 12th International Conference on Flow Dynamics, ICFD2015, October 2015

10. T. Ambo, et. al., "Support Interference Effects on Aerodynamic Forces of a Magnetically Suspended 6:1 Proplate Spheroid Model", 12th International Conference on Flow Dynamics, ICFD2015, October 2015

11. D-K. Lee, et. al.: "Dynamic Calibration of Magnetic Suspension and Balance System for Sting-Free Measurements in Wind Tunnel Tests", Journal of Mechanical Science and Technology 27(7), 2013, pp:1963-1970

12. A.K. Owen, F.K. Owen, "Magnetic Suspension and Balance Testing in Support of Hyper-X", 12th AIAA International Space Planes and Hypersonic Systems and Technologies Conference, December 2003. AIAA 2003-6958

13. M. Judd, "The Magnetic Suspension and Balance System as a Wind Tunnel Dynamic Balance", $3^{\text {rd }}$ International Congress on Instrumentation in Aerospace Simulation Facilities, ICIASF'69, May 1969, pp:198-206

14. T. Stephens, et. al., "Recent Developments in a Wind Tunnel Magnetic Balance", $10^{\text {th }}$ AIAA Aerospace Sciences Meeting, January 1972.

15. S. Abdel-Kawi, et. al. "Aerodynamic Data Acquisition with the University of Southampton Magnetic Balance", $2^{\text {nd }}$ International Symposium on Electro-Magnetic Suspension, July 1971

16. C. Haldeman, et. al. "Improvements in the Magnetic Suspension and Balance System Required for Magnus Testing", $5^{\text {th }}$ International Congress on Instrumentation in Aerospace Simulation Facilities- ICIASF'73, September 1973

17. R. Oshima, H. Sawada, S. Obayashi, "A Development of Dynamic Wind Tunnel Test Techniques by Using a Magnetic Suspension and Balance System", 54th AIAA Aerospace Sciences Meeting, January 2016. AIAA 2016-1541

18. E. Murman, "Experimental Studies of a Laminar Hypersonic Cone Wake", AIAA Journal, Vol. 7, No. 9, September 1969, pp:1724-1730

19. H.H. Parker, R.N. Zapata, "The University of Virginia Cold Magnetic Wind Tunnel Balance", $8^{\text {th }}$ Navy Symposium on Aeroballistics, June 1969, pp:695-716

20. C.P. Britcher, "Feasibility of Dynamic Stability Measurements of Planetary Entry Capsules Using MSBS", 12th International Conference on Flow Dynamics, ICFD2015, October 2015

21. T. Stephens, "Design, Construction and Evaluation of a Magnetic Suspension and Balance Systems for Wind Tunnels", NASA CR-66903, November 1969.

22. T. Schott, T. Jordan, T. Daniels, "Present Status of the MIT/NASA Langley 6-inch MSBS", International Symposium on Magnetic Suspension Technology, NASA CP-3152, August 1991

23. P. Tcheng, T. Schott, "A Five Component Electro-Optical Positioning System, $12^{\text {th }}$ International Conference on Instrumentation in Aerospace Simulation Facilities, ICIASF'87, June 1987

24. T. Stephens, "An Electromagnetic Remote Model Position Sensing System for Wind Tunnels with Particular Application to Magnetic Suspension Systems", $2^{\text {nd }}$ International Symposium on Electro-Magnetic Suspension, July 1971

25. T.S. Daniels, J.S. Tripp, "Improvements to an Electromagnetic Position Sensor for a Magnetic Suspension Wind Tunnel", 1988. ISA 88-0708

26. Britcher, C.P; Johnson, D.: "Feasibility of Dynamic Stability Measurements of Planetary Entry Capsules Using a Magnetic Suspension and Balance System". AIAA Aviation, June 13-17, 2016, AIAA 2016-4162

27. Johnson, D.: "Modeling of a Wind Tunnel Magnetic Suspension and Balance System". AIAA Region I student conference, April 2017

28. Barlow, J.B.; Rae, W.H.; Pope, A.: Low-Speed Wind Tunnel Testing. 3rd edition, Wiley, 1999

29. Mikhailov, M.D.; Silva Freire, A.P.: "The Drag Coefficient of a Sphere: An Approximation using Shanks Transform". Poweder Technology, 237 (2013), pp:432-435

30. Roos, F.W.; Willmarth, W.W.: "Some Experimental Results on Sphere and Disc Drag". AIAA Journal, 9 (1971), pp:285-290

31. R. Oshima, et. al;., "Dynamic Calibration of Magnetic Suspension and Balance System for Aerodynamic Force Measurement", 12th International Conference on Flow Dynamics, ICFD2015, October 2015 